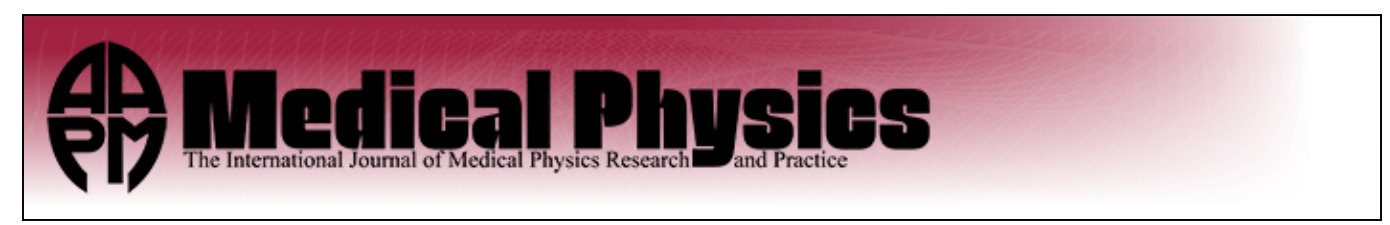

\title{
Accurate calibration method for 3D freehand ultrasound probe using virtual plane
}

Danilo De Lorenzo, Alberto Vaccarella, Ghassan Khreis, Holger Moennich, Giancarlo Ferrigno, and Elena De Momi

Citation: Medical Physics 38, 6710 (2011); doi: 10.1118/1.3663674

View online: http://dx.doi.org/10.1118/1.3663674

View Table of Contents: http://scitation.aip.org/content/aapm/journal/medphys/38/12?ver=pdfcov

Published by the American Association of Physicists in Medicine

\section{Articles you may be interested in}

Impact of SPECT corrections on 3D-dosimetry for liver transarterial radioembolization using the patient relative calibration methodology

Med. Phys. 43, 4053 (2016); 10.1118/1.4953203

Calibration of coronary calcium scores determined using iterative image reconstruction (AIDR 3D) at 120, 100, and $80 \mathrm{kVp}$

Med. Phys. 43, 1921 (2016); 10.1118/1.4942484

Rapid 3D human ribcage and kidney modeling for transcostal HIFU surgery

AIP Conf. Proc. 1503, 95 (2012); 10.1063/1.4769924

Validation of 3D reconstructions of a mimicked femoral artery with an ultrasound imaging robotic system Med. Phys. 37, 3868 (2010); 10.1118/1.3447721

Development of a three-dimensional freehand endorectal ultrasound system for use in rectal cancer imaging Med. Phys. 32, 1757 (2005); 10.1118/1.1925228

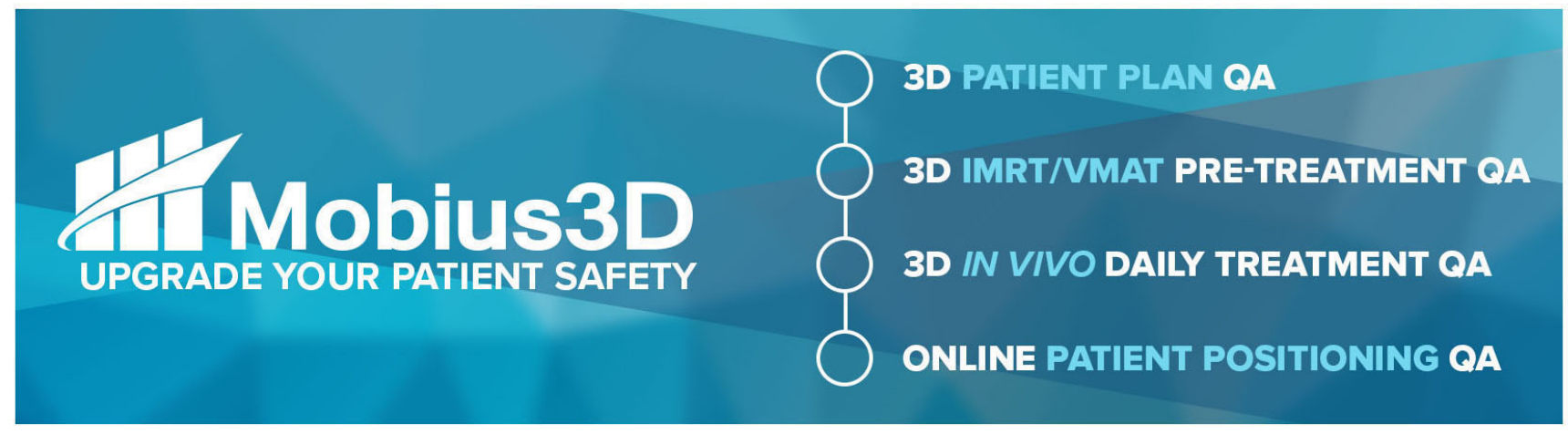




\title{
Accurate calibration method for 3D freehand ultrasound probe using virtual plane
}

\author{
Danilo De Lorenzo, Alberto Vaccarella, ${ }^{\text {a) }}$ and Ghassan Khreis \\ Politecnico di Milano, Bioengineering Department, Neuroengineering and Medical Robotics Laboratory, \\ Milano 20133, Italy \\ Holger Moennich \\ Karlsruhe Institute of Technology, Institute for Process Control and Robotics, Karlsruhe D-76131, Germany \\ Giancarlo Ferrigno \\ Politecnico di Milano, Bioengineering Department, Neuroengineering and Medical Robotics Laboratory, \\ Milano 20133, Italy \\ Elena De Momi \\ Politecnico di Milano, Bioengineering Department, Neuroengineering and Medical Robotics Laboratory, \\ Milano 20133, Italy and Istituto di Tecnologie Industriali ed Automazione, Consiglio Nazionale delle Ricerche, \\ Milano 20133, Italy
}

(Received 3 March 2011; revised 3 November 2011; accepted for publication 4 November 2011; published 29 November 2011)

Purpose: In this paper a new, easy-to-manufacture and easy-to-use ultrasound (US) probe calibration phantom for 3D freehand scanning is presented and evaluated, together with a new method for achieving an accurate and user-robust calibration using virtual plane.

Methods: The phantom allows the optically tracked US probe to perform two rotations and two translations while keeping the image of a tensioned wire in the image plane. This approach allows obtaining a sharp image of the wire independently from the probe pose. The virtual plane allows the calibration algorithm to converge minimizing the required number of US probe tracked poses. The US image and position data are synchronized via a CORBA interface, created within the Image Guided Surgery Toolkit (IGSTK) framework. The calibration algorithm and the calibration protocol were evaluated in a set of experiments carried out by different test-users.

Results: The calibration method proved to be accurate and precise: 3D point reconstruction accuracy resulted $0.2 \mathrm{~mm}$ as mean value, while the precision was $0.4 \mathrm{~mm}$ as standard deviation.

Conclusions: The technique showed to be suitable for medical applications from morphological diagnosis to intraoperative surgical planning adaption. (C) 2011 American Association of Physicists in Medicine. [DOI: 10.1118/1.3663674]

Key words: freehand 3D ultrasound, US probe calibration, surgical navigation, intraoperative planning

\section{INTRODUCTION}

Three-dimensional (3D) Ultrasound (US) imaging is a noninvasive medical diagnostic method. Although being generally known for qualitative assessment of fetal morphology, its importance for intraoperative imaging in surgical procedures, where organs or soft tissues morphology varies with respect to the preoperative situation, is rapidly increasing. In neurosurgery, for example, US images of the brain are registered with preoperative magnetic resonance imaging (MRI) data to detect the brain shift occurring during the operation. ${ }^{1-4}$ In radiotherapy, 3D US imaging is used to update the radiation dose delivery plan with respect to preoperative treatment planning, which is based on CT images. ${ }^{5,6}$ US imaging has also been used for intraoperative registration of bone segments during orthopedic surgery ${ }^{7}$ and for abdominal surgery. ${ }^{8,9}$

\section{I.A. Spatial calibration}

3D ultrasound imaging can be built from 2D images by tracking the position of a probe in space, by a mechanical, optical or electromagnetic localization system. 3D US based on feature matching ${ }^{10}$ is also possible, but it is unsuitable for intraoperative applications, due to poor accuracy. Spatial localization systems measure the pose of a rigid body solid with the ultrasound probe, hereafter referred to as the "dynamic reference frame" (DRF). The probe calibration process determines the transformation matrix between the coordinate frame of the probe DRF and the B-scan plane. ${ }^{11}$ In this process, phantoms with known geometry are used in order to identify features in both the US image and in the physical phantom space and spatial relationship is therefore established. ${ }^{11}$ Manual selection of the features and automatic image segmentation techniques are used for identifying the phantom features in the US images. ${ }^{11,12}$ Images are distorted since the speed of sound of the coupling medium is different and not constant (in depth and in time) from the one assumed inside the ultrasound device $(1540 \mathrm{~m} / \mathrm{s}$ is considered as the mean speed of US in human soft tissues). Calibrating the US probe means estimating the unknown transformation matrices involved in the spatial relationship between the US 
image space and the localization device space. A closed form solution can be computed if the position of the phantom features are known exactly in the US image and in the physical reference frames, otherwise an iterative approach is necessary. ${ }^{11,13}$ Phantoms can be made of fiducial points, ${ }^{13-15}$ cross-wires, ${ }^{16,17} \mathrm{Z}$ shaped wire, ${ }^{18}$ and planes (wall phantoms). ${ }^{12,19,20}$ Methods based on points features require the alignment of the B-scan with the target point and the set of the optimized calibration parameters can get stuck in local minima. $^{12}$ On the contrary, $\mathrm{Z}$ shaped phantoms do not require any alignment of the image plane with the phantoms geometrical feature, but they need an accurate mechanical machining and an accurate phantom calibration to know the exact position of the wires. The deformation of $\mathrm{Z}$ wires, due to the wearing effect of time, is difficult to compensate for. The accuracy of such calibration methods remain poorer than other phantoms. ${ }^{15}$ In case of wall phantoms, the probe calibration procedure estimates 11 unknown parameters: 6 for the transformation matrix from the probe DRF to the reference frame solid with one corner of the B-scan image plane, 3 for the definition of the plane (wall) and 2 parameters for the horizontal and vertical scales (for converting pixels to millimeter) in the B-scan image.

Prager et al. ${ }^{12}$ were the first to scan a planar surface ("single-wall phantom") instead of target points. Lango 19 made the same by using a nylon membrane, while Rousseau et $a .^{20}$ used a Polymethylmethacrylate plate. A plane appears as a set of straight lines in the US B-scans, one for each data frame, thus using a flat surface immersed in a water tank allows achieving more redundancy than a singlepoint calibration, and more reliable, potentially automatic, feature segmentation. However, when the US plane is not normal to the wall, much of the US energy is reflected away from the probe ${ }^{12}$ and the first echo that returns back to the probe comes from the edge of the beam closest to the wall. ${ }^{12,19,21}$ Therefore, the detected line thickness is not uniform with respect to different probe poses.

A further enhancement to the single-wall phantom, circumventing the latter problem, is the Cambridge phantom, ${ }^{12}$ which scans a bar that moves with wheels on a flat surface. Nevertheless, it is not easy to manipulate and, at high depths, the probe clamp reverberation reduces the quality of the images. ${ }^{11}$ The scanning protocol demands for considerable user expertise to ensure that the calibration optimization is not under-determined. ${ }^{14}$ Incorrect calibration caused by incomplete scanning of the phantom is thus by far the most common source of error. Gee et al. ${ }^{22}$ proposed another calibration phantom, where the position sensor is replaced by mechanical guides, but it requires accurate machining and is quite complex to be built. Hsu et al. ${ }^{23}$ demonstrated that even if some movements (translation along the $\mathrm{X}$ and the $\mathrm{Y}$ and rotation about the $Z$ axis of the probe) are missing during the calibration, the solution of the optimization process is well-constrained. The same author provided indications for the minimum set of scanning patterns required for a planebased calibration. ${ }^{24}$ Problems in the identification of the line representing the intersection between the image and the wall phantom were solved in Hartov et al., ${ }^{25}$ where an adaptive algorithm was used to eliminate data that would otherwise prevent the convergence of the procedure. The algorithm proved to be robust, but accuracy was unsatisfactory for neurosurgical applications.

\section{I.B. Synchronization}

The DRF position and the ultrasound image data are time-stamped by the computer with an unknown delay after being detected by the hardware. ${ }^{26,27}$ Synchronization requires finding the latency between the position sensor and ultrasound image timestamps. Jacobs et al. ${ }^{28}$ showed that the latency estimation is a crucial problem. In their work, the measured latency was $220 \mathrm{~ms}$ for images gathered at $10 \mathrm{~Hz}$. Rousseau et $a .^{20}$ proposed a new approach for temporal calibration of 3D ultrasound systems which does not introduce any constraints on probe motion. Latency estimation is stated as a robust minimization problem. Experiments showed that accurate latency estimation is preferably conducted using "harmonic" data sets. Hsu et al. ${ }^{29}$ designed a phantom based on overlaying membranes in order to calibrate the temporal offset relying on the segmentation reliability, on the phantom construction tolerances and on spatial calibration accuracy.

\section{I.C. Innovation}

The original Cambridge phantom ${ }^{12}$ and its subsequent improvements ${ }^{15,22-24,29}$ allows the simultaneous movements of the probe around a bar and translation on a flat surface on wheels. The phantom displacement on the plane does not add any information on the transformation between the probe DRF reference frame and the US image plane reference frame. Translating the probe parallel to the plane (2 degrees of freedom, DoFs), the same US image is acquired. Therefore, in this paper a simplified new plane-based phantom (the "NearLab phantom") is presented. The phantom is both easy to manufacture and to manipulate in order to achieve an accurate and precise calibration. ${ }^{24}$ The main innovation of our calibration method is that a physical plane is not used for the calibration. Translations of the phantom on the wall are simulated (giving rise to a virtual displacement on a "virtual plane") in order to well condition the optimization approach. ${ }^{12}$ Well conditioning of the, almost standard, optimization approach, given by planar translations in the Cambridge phantom, is therefore preserved. The imaged geometry (a line, instead of a plane) is very thin and sharp and its image does not change while moving the probe around the wire line (4 DoFs). The imaged wire is easy to be tensioned in order to prevent deformations due to machining and/or wearing that affect other more complex phantom structures. Alignment of the imaged wire in the image plane is also achieved easily due to the adjustability of the supporting frame. No additional measurement instruments are needed since the US image qualitative inspection is already adequate to determine the correct alignment of the wire in the image plane.

Synchronization is guaranteed by a client-server framework using the CORBA communication protocol designed on purpose. 
The paper is organized as follows: in the first part we present the system framework, which is part of the ROBOCAST project. ${ }^{30}$ Then the spatial calibration formulation and the optimization used by the new method are reported together with the NearLab phantom design. A set of experiments was designed in order to test spatial and temporal calibration performances also under user variability. Results are presented and discussed.

\section{MATERIALS AND METHODS}

\section{II.A. The imaging framework}

The 3D imaging architecture encompasses (as shown in Fig. 1):

- An ultrasound console (ProSound Alpha 7, ALOKA Ltd, Japan).

- An ultrasound burr-hole probe (ALOKA UST-52114P, 3-7.5 MHz with phased array transducer).

- An optical tracking system (Optotrack Certus NDI, Waterloo, Ontario, Canada), with $0.15 \mathrm{~mm}$ accuracy.

- A custom built DRF with four active markers, attached to the probe.

- A Sensor Manager framework $(\mathrm{SM})^{31}$ running on a dual Core laptop with 2 GB RAM, Windows XP platform.

- An S-video frame grabber (EZ Grabber, PAL, $720 \times 576$ $\mathrm{px}, 25 \mathrm{~Hz})$.

The calibration and visualization modules run on a computer with Intel ${ }^{\circledR}$ Core $^{\mathrm{TM}_{2}}$ Quad Processor Q9550 (12M Cache, $2.83 \mathrm{GHz}, 1333 \mathrm{MHz}$ FSB), Windows XP platform. The algorithms to solve the calibration optimization problem were developed in $\mathrm{C}++$ and MATLAB 2008a (Mathworks, Inc., Natick, MA).

The US console and the optical tracking system are connected to the SM via the Frame Grabber and via Ethernet connection respectively. The ROBOCAST system architec- ture is based on a client-server model, as shown in Fig. 2. All the sensors are connected to the SM application, based on the IGSTK and on the OpenCV libraries, which provide sensor data to one or more dependent modules (clients). The system can asynchronously provide images and tracking data to clients connected via Ethernet. In the ROBOCAST application, the calibration module runs on a client connected to the SM via TAO-CORBA middle-ware. ${ }^{32,33}$ The SM ensures temporal alignment among tracking sensor systems by storing each position data in a transform object which also provides a time stamp and a validity time window. In this way it is possible to verify if data coming from different tracking sensors were acquired at the same time instant (within a user defined threshold).

The freehand 3D US systems indeed require a stream of position data and images captured at the same instant, however when the client receives an image and a DRF position after a request, they could have different time-stamp, depending on the latencies of the two data streams:

- the latency of the tracking system;

- the latency of the US imaging system;

- the latency between the client request and the SM response.

The first two latencies are due to the delay between the sensor data sending and the SM data receiving. The latency between the SM and the clients depends on the number of clients requesting sensor data and on the rate of the requests.

\section{II.B. The NearLab calibration phantom}

The calibration phantom designed in this work encompasses three aluminium bars and a U-shape probe support with slides (Fig. 3). One bar lies at the bottom of the phantom and two vertical bars, with sliding guides, are attached

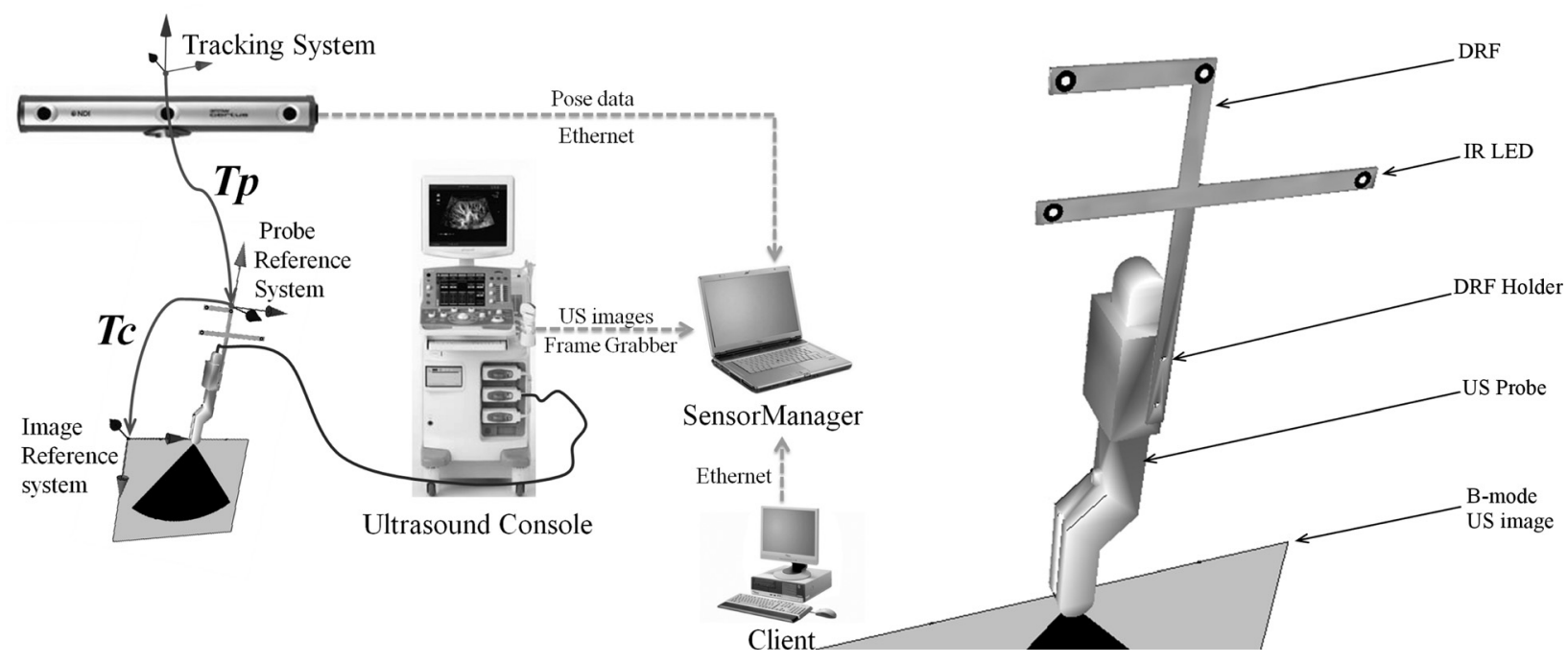

FIG. 1. On the left, the imaging system and the sub-modules connections. $T_{p}$ is the localisation matrix of the probe DRF provided by the tracking system, $T_{c}$ is the probe calibration matrix. The SM receives tracking data via Ethernet connection and images via a Frame Grabber. Client communication is assured by Ethernet connection. On the right, the probe DRF. The DRF is attached to the probe through a holder. 


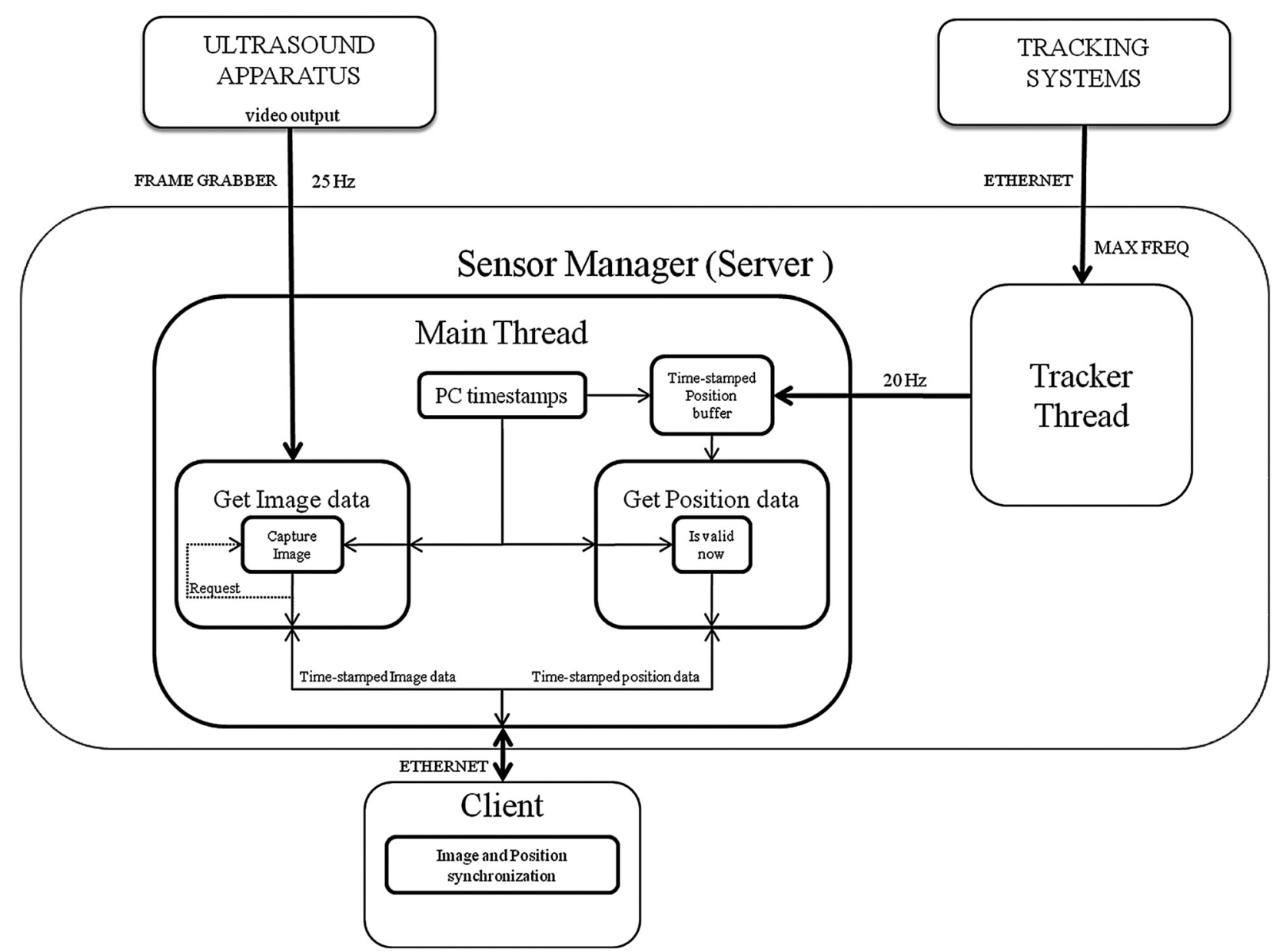

FIG. 2. Position data are managed by the Sensor Manager. Each tracking system is connected to a separate thread (Tracker Thread) that acquires position data at the tracking system maximum allowed frequency (6400/N for the Certus optical tracking system, with $N$ equals to the number of active infrared markers). The Main Thread in the SM block acquires the position data at $25 \mathrm{~Hz}$ (user-set frequency) from the Tracking Thread into a circular buffer and tags each data with a timestamp representing the instant of capturing. When a Client requests a position data, the GetPosition module verifies that the newest data are still valid and then sends it to the client. The Get Image data module captures a B-scan image via OpenCV. Each captured image is tagged with a timestamp.

to it. The vertical bars, rigidly connected together via the probe support, can rotate with respect to the bottom bar around a steel wire covered with nylon $(0.45 \mathrm{~mm}$ in diameter), and tensioned using two hollow screws $(0.5 \mathrm{~mm}$ diameter) (Front to Back Rotation around A axis, as shown in Fig. $3)$. The probe support also has two possible translations along the two vertical bars (vertical translation, $Z$ axis) and along the horizontal bar (lateral translation, $X$ axis). A DRF is attached to the probe through a holder. The probe holder has a concavity matching the probe handle. It adds two further degrees of freedom to those of probe support (see above), it can rotate around it, i.e., around the $Y$ axis of the probe (Side to Side Rotation), and can translate along the U-shape bar ( $Y$ translation) during the phantom setup phase. A screw driver is used to fix the position and the orientation of the bar and of the probe support.

The bars and the probe holder were manufactured in the workshop of ITIA CNR in Milan, Italy.

\section{II.C. The calibration algorithm}

The calibration problem can be stated as follows. Given the spatial transformations relationship: ${ }^{11}$

$$
\left[\begin{array}{c}
P_{x}^{\text {Plane }} \\
P_{y}^{\text {Plane }} \\
P_{z}^{\text {Plane }} \\
1
\end{array}\right]=T_{W}^{-1} \cdot T_{P} \cdot T_{C} \cdot\left[\begin{array}{c}
S_{x} \cdot P_{x}^{I} \\
S_{y} \cdot P_{y}^{I} \\
0 \\
1
\end{array}\right]
$$

"calibrating" means finding the transformation $T_{C}$ between the US-image plane and the probe DRF $\left(T_{p}\right.$, as shown in Fig. 4), given:

- $T_{P}$, i.e., the transformation between the probe DRF reference frame and the tracking system (global) reference frame;

- $T_{W}$, i.e., the transformation between the tracking reference system and the phantom (virtual plane) reference system;

- $P^{I}$, i.e., a point on the US image, belonging to the segmented line;

- $S_{x}$ and $S_{y}$, i.e., the image scale factors;

$-P^{\text {Plane }}$, i.e., $P^{I}$ expressed in the plane reference frame.

The image scale factors $\left(S_{x}\right.$ and $\left.S_{y}\right)$ were both determined using the distance measurement tool of the ultrasound machine, as indicated in Hsu et al. ${ }^{23}$ Temperature correction factor was applied to the features in the US images, as 


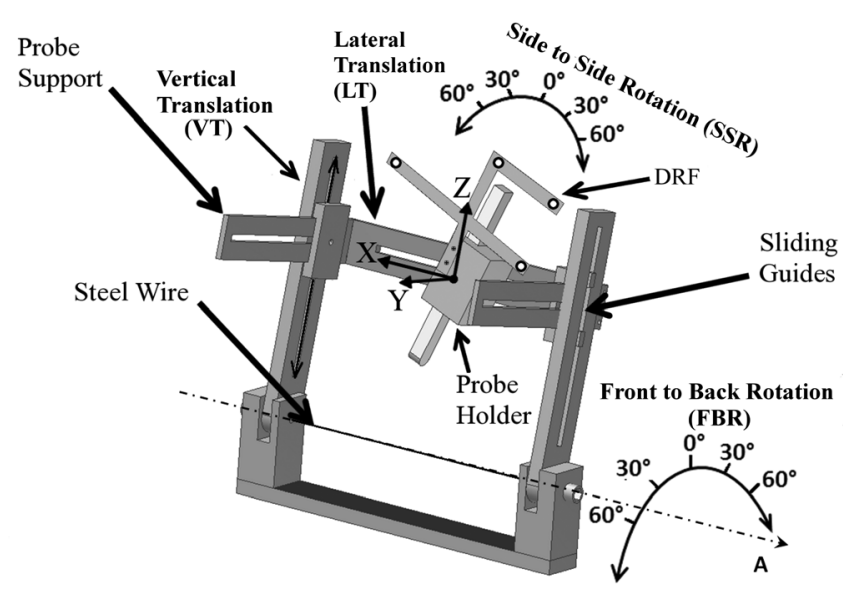

FIG. 3. The NearLab phantom degrees of freedom. The tensioned wire is also visible at the bottom. On the probe, the movement reference system is shown: translation along the $Y$ axis is used during the phantom setting procedure, in order to align the probe image plane with the steel wire. Translations along the $Z$ axis are referred as vertical translation (VT) and translation along the $X$ axis are referred as lateral translation (LT).

suggested in Hsu et al. ${ }^{23}$ in order to remove any error due to the sound speed variations. Image segmentation was performed in the user defined region of the ultrasound image using the RANSAC line detection algorithm. ${ }^{24}$

The nine unknown parameters ( 6 parameters of the calibration transformation, $T_{C}$, and 3 parameters describing the phantom plane, which are not used during the probe tracking) are determined minimizing the Euclidean distance $\left(D_{i}\right)$ between the points lying on the segmented lines in the US images $\left(P^{I}\right)$, transformed $\left(\hat{P}^{\text {Plane }}\right)$ using the actual estimated calibration transformation $\left(\hat{T}_{C}\right)$, and the virtual phantom plane $\left(a x+b y+c z+d=0\right.$, and $\left.\sqrt{a^{2}+b^{2}+c^{2}}=1\right)$. The transformed points in the virtual plane reference frame are:

$$
\left[\begin{array}{c}
\hat{P}_{x}^{\text {Plane }} \\
\hat{P}_{y}^{\text {Plane }} \\
\hat{P}_{z}^{\text {Plane }} \\
1
\end{array}\right]=T_{W}^{-1} \cdot T_{P} \cdot \hat{T}_{C} \cdot\left[\begin{array}{c}
S_{x} \cdot P_{x}^{I} \\
S_{y} \cdot P_{y}^{I} \\
0 \\
1
\end{array}\right]
$$

The function to be minimized is:

$$
f=\min _{f} \frac{1}{2} \sum_{i=1}^{N} D_{i}^{2}
$$

where

$$
D_{i}=a x_{i}+b y_{i}+c z_{i}+d
$$

and $\left[x_{i}, y_{i}, z_{i}\right]$ are the $3 \mathrm{D}$ coordinates of the $i$-th transformed point $\left(\hat{P}^{\text {Plane }}\right) . N$ is the total number of measurements.

For the iterative optimization, the LevenbergMarquardt ${ }^{11,35}$ algorithm, which combines the characteristics of both Gradient Descent and Gauss-Newton methods, was used. The phantom plane, identified by $T_{w}$ is any virtual plane passing through the steel wire. A series of simulated translations along the $X$ and $Y$ axis of the probe DRF were also used to increase the calibration algorithm robustness, virtually "painting" the plane (virtual) with a series of lines, as done by the other approaches, ${ }^{12}$ although in this case no

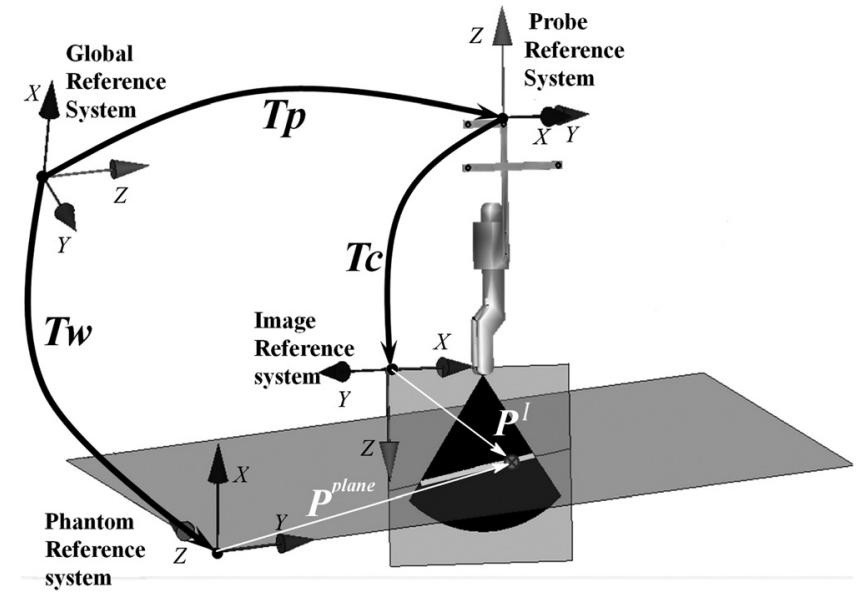

FIG. 4. The spatial transformations involved in the probe calibration problem. $T_{P}$, is the transformation between the probe DRF reference frame and the tracking system reference frame; $T_{W}$, is the transformation between the tracking reference system and the phantom (virtual plane) reference system; $T_{C}$, is the calibration transformation between the US-image plane and the probe DRF.

physical movements are accomplished. In order to fix one of the infinite number of planes passing through the steel wire, the plane normal was chosen equal to the $Z$-axis versor of the first acquired probe reference frame. Virtual translations guarantee that the virtual plane will not change too much (theoretically it should not change substantially, but the presence of noise gives raise to small variations) during the iterations. Using this constraint, the optimization converges more robustly to a global minimum, since the unknown plane parameters are less sensitive to noise.

A user friendly graphical interface was designed in order to facilitate the calibration steps (Fig. 5).

\section{II.D. Experimental protocol}

The experimental setup is shown in Fig. 6. In order to assess the NearLab phantom (NP) performances in terms of spatial calibration accuracy and precision, ${ }^{11}$ the following protocol was followed (see Table I for details):

- Calibration method. All calibrations were performed using the NearLab phantom

1. using the calibration method described in the following "calibration protocol" paragraph (NP);

2. using temperature correction factor $(\mathrm{NP}+\mathrm{TC})$;

3 . as 1 and 2, adding virtual translations: 4 lateral translations in $Y$ direction ( $5 \mathrm{~cm}$ spaced) and 4 translations (5 cm spaced) along $X$ direction (Fig. 3) $(\mathrm{NP}+\mathrm{TC}+\mathrm{S})$.

Each calibration was repeated 8 times.

- calibration protocol. Calibration was performed using the NearLab phantom with the following probe movements (Fig. 3):

1. 10 Front to Back Rotations of the probe (FBR);

2. As above plus 10 Side to Side Rotations of the probe $(\mathrm{FBR}+\mathrm{SSR})$; 


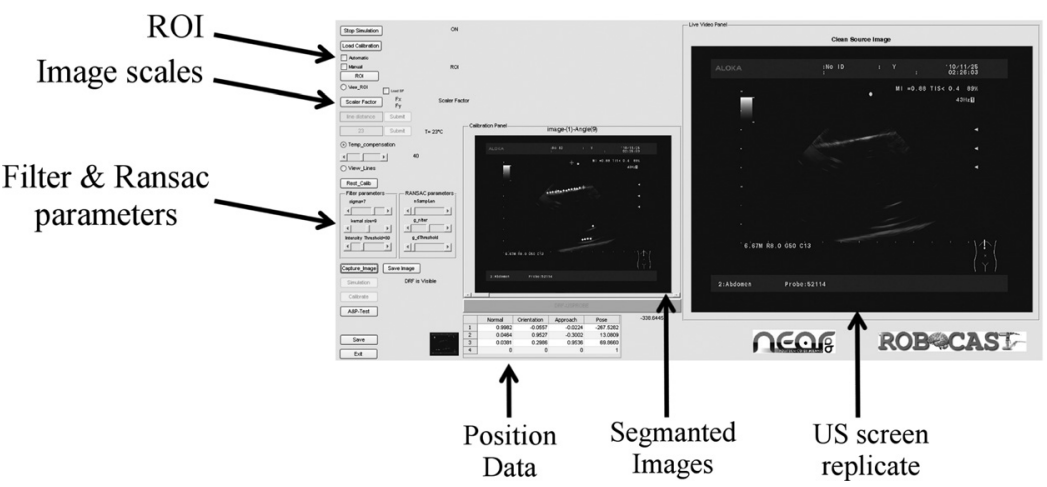

FIG. 5. The developed GUI for probe automatic calibration: buttons on the left allow the user to set the calibration parameters, the region of interest (ROI) on the ultrasound image, the scale factors and the temperature correction factor. Calibration performances are also displayed. In the central panel, segmented images are shown and probe visibility is represented as a green strip. On the right, the US screen is replicated.

3. As above plus 10 Vertical Translations (translations along $Z$ direction) (FBR + SSR + VT);

4. As above plus 10 Lateral Translations (translations along $X$ direction) $(\mathrm{FBR}+\mathrm{SSR}+\mathrm{VT}+\mathrm{LT})$.

Each calibration was repeated 5 times.

- Intersubject and intrasubject variability: In order to test the calibration robustness, performances were compared with the Classical Free-hand Calibration (CFC) ("Membrane phantom") ${ }^{19}$ asking 4 non experienced users to perform 5 CFC and 5 NearLab phantom-assisted ("NearLab phantom") calibrations. CFC was performed asking the users to change the probe position 40 times in a water tank with a planar membrane in the bottom, changing the configuration of a mechanical linkage that held the probe still while the DRF poses were acquired and averaged. ${ }^{36}$

In the experiments, the water temperature was monitored constantly using a digital multimeter (IDM-62T, ISO-tech, United Kingdom).

\section{II.E. Evaluation metrics}

For both the experiments, the accuracy and precision ${ }^{14}$ were evaluated using a Plexiglas ${ }^{\circledR}(300 \times 200 \times 10 \mathrm{~mm})$ plane as the object test to be reconstructed. The plane was

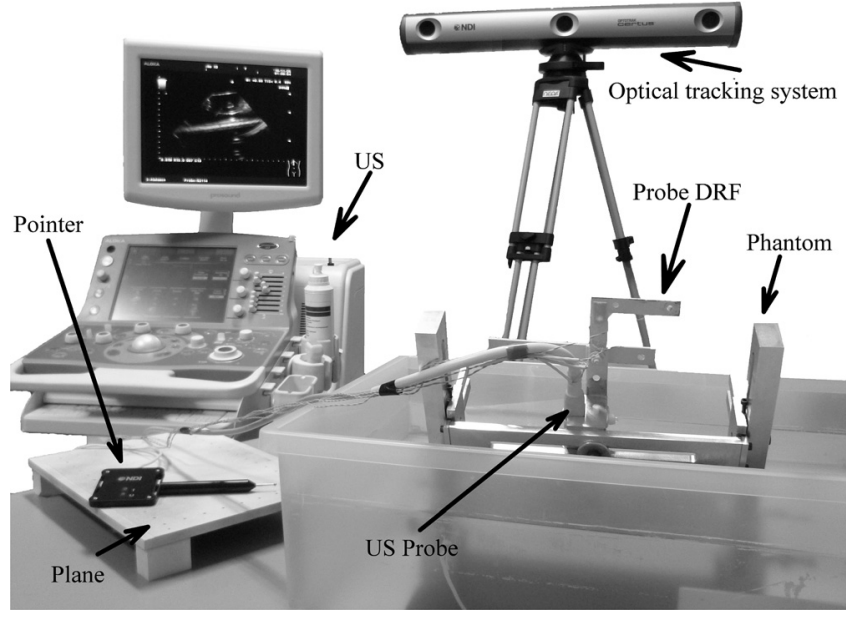

FIG. 6. The experimental setup. On the back the optical tracking localization system is visible, on the left the US images acquisition system is shown. The calibration object and the probe are immersed in water. immersed and fixed in the water tank and localized using an optical pointer (NDI, Waterloo, Ontario, Canada). Equidistant divots (42) with the same diameter of the pointer tool ( $3 \mathrm{~mm}$ ) were machined on the plane with $0.15 \mathrm{~mm}$ accuracy. Given the 42 points 3D coordinates, the plane equation was computed with a least square approach (MATLAB R2008a).

For each calibration performed, 5 testing datasets were considered, each dataset composed of 40 images of $80 \mathrm{seg}$ mented points each.

The following error measures were computed:

- Plane accuracy $\left(A_{i}\right)$ : Computed as the distance between the plane, and the points reconstructed using the 3D US system.

$A_{i}=\left\|P_{i}^{\text {Plane }}-P_{i}^{\text {plexPlane }}\right\|$

where $P_{i}^{\text {plexplane }}$ is the projection of $P_{i}^{\text {Plane }}$ on the Plexiglas ${ }^{\circledR}$ plane.

- Plane precision $\left(P_{i}\right)$ : Computed as the distance between the "mean plane" (i.e. the one minimizing the sum of the squared distances from reconstructed points) and the points reconstructed using the 3D US system.

$P_{i}=\left\|P_{i}^{\text {Plane }}-P_{i}^{\text {MPlane }}\right\|$

where $P_{i}^{\text {MPlane }}$ is the projection of $P_{i}^{\text {Plane }}$ on the mean plane.

TABLE I. Experimental protocol.

\begin{tabular}{lccc}
\hline \hline & $\begin{array}{c}\text { Number } \\
\text { of } \\
\text { calibrations }\end{array}$ & $\begin{array}{c}\text { Tests dataset } \\
\text { for each } \\
\text { calibration }\end{array}$ & $\begin{array}{c}\text { Number } \\
\text { of probe } \\
\text { poses }\end{array}$ \\
\hline Calibration method & 8 & - & 40 \\
NP & - & 5 & 40 \\
NP + TC & - & - & \\
NP + TC + S & - & - & 40 \\
Calibration protocol & - & 5 & 10 \\
FBR & - & - & 30 \\
FBR + SSR & - & - & 40 \\
FBR + SSR + VT & 5 & - & - \\
FBR + SSR + VT + LT & - & 5 & 40 \\
Inter/Intra subject variability & & & \\
Membrane phantom & & & \\
NearLab phantom & & & \\
\hline
\end{tabular}


As stated by several authors, ${ }^{4,12,18,41} A_{i}$ is commonly referred to as point reconstruction accuracy, while $P_{i}$ as point reconstruction precision.

Distance measures are signed, in order to differentiate whether line points are estimated above or below the estimated plane. Signed distances of points above the plane are given a positive sign, those below a negative one. Data population proved to be normally distributed (Lilliefors test for normality with $p<0.05$ ) and therefore the mean and the standard deviation were computed as distribution parameters. Statistical significant variance difference was assessed using the Fisher test, with $p<0.05$ (Statistica 7, Stat Soft).

\section{II.F. Temporal offset}

In order to estimate the temporal offset between the DRF tracking data and the US image, the probe immersed in the water tank was moved along the vertical direction ( $Z$ axis of Fig. 4) using a 6 DoF serial robot (Pathfinder, Prosurgics, United Kingdom) which was programmed to achieve three repetitions of vertical motion (lasting $0.83 \mathrm{~s}$ at $30 \mathrm{~mm} / \mathrm{s}$ maximum velocity).

Position data of a point in the US image (the line) and of the probe DRF were acquired and normalized with respect to the amplitude of the movement performed. Signal alignment was done using the timestamps provided by the SM framework and the time lag was estimated by computing the maximum of the cross correlation function of the two time series.

\section{RESULTS}

\section{III.A. Spatial calibration}

As shown in Fig. 7, both the temperature correction factor (TC-during the acquisitions the water temperature varied between 21 and $23^{\circ} \mathrm{C}$ ) and the simulated (S) translation allow decreasing the polarization above the actual plane (from 1 to $0.2 \mathrm{~mm}$ as mean value). Adding simulated movements allows also decreasing the error standard deviation: $68.2 \%$ of the data population is bounded below $0.6 \mathrm{~mm}$. When estimating the precision, the difference in the variances is not statistically significant.

Figure 8 shows the distance errors with respect to the probe movements executed. Only in case of FBR the distance error is biased with respect the actual plane (mean error is $1.581 \mathrm{~mm}$ ). Adding the SSR reduces the mean error to $0.226 \mathrm{~mm}$ and adding the VT further reduces the error to $0.106 \mathrm{~mm}$.

In terms of standard deviation, each movement allows reducing the standard deviation, apart from LT which did not improve the calibration performance (in $\mathrm{FBR}+\mathrm{SSR}+\mathrm{VT}$ the standard deviation is $0.39 \mathrm{~mm}$ while in $\mathrm{FBR}+\mathrm{SSR}+\mathrm{VT}+\mathrm{LT}$ the standard deviation is 0.41 $\mathrm{mm}$ ). Adding the LT, does not change the standard deviation when computing the calibration precision.

Figure 9 shows the distance errors (accuracy $A_{i}$ and precision $P_{i}$ ) with respect to the calibration method and with respect to the user. Using the Membrane phantom the average accuracy error is around $1 \mathrm{~mm}$ as mean value for User 3 (worst case), while using the NearLab phantom the polarization decreases to $0.2 \mathrm{~mm}$ as mean value for User 3 . In terms of standard deviation, the performances obtained with the NearLab phantom are significantly different from those of the Membrane phantom. Similar performances are obtained when evaluating the precision of the calibration $(0.4 \mathrm{~mm})$.

\section{III.B. Synchronization}

As shown in Fig. 10, the time lag between the DRF data and the US image resulted in a median value of $230 \mathrm{~ms}$ (223 and $240 \mathrm{~ms}$ the interquartile range).

\section{DISCUSSION}

Intraoperative ultrasound images acquisition is becoming a more and more widespread technique for updating the preoperative plan during the surgical interventions and represents a cost-effective alternative to intraoperative MR. ${ }^{37}$ In particular, in neurosurgery, the intraoperative brain morphology can be different with respect to the preoperative plan: the more the skull is open, the more the brain shifts, due to both deliquoration and pressure variation increase. ${ }^{38}$ US images should be accurately registered on MR images to quantify the brain shift amount and to accurately localize deep brain structures.

Probe calibration must be repeated at long or short time intervals if the DRF is not permanently attached. Adapter cannot guarantee perfect repeatability, so calibration is needed often. Therefore, the need for an easy to use and easy to manufacture phantom for freehand 3D ultrasound arises. In this paper, we presented and evaluated the performances of the NearLab phantom, which is extremely easy-to-replicate and easy-to-use, due to the limited number of pieces to be assembled, to the probe movements allowed and to the small size. The only difficulty that may be incurred in by the users is aligning the US probe scanning plane with the steel wire; however the user can use wedges ${ }^{22}$ in order to easily carry out this step. After fixing the probe support to the handles, only four degrees of freedom are left, those have been shown to be sufficient to perform an accurate calibration. The user can manipulate easily the handles and the probe holder following a specific protocol. This phantom is a "virtual plane"-based phantom where a tensioned steel wire in the image plane gets rid of the beam thickness problem: all the obtained images are of good quality at all angles and positions allowed by the phantom. Good quality images increase the reliability of the calibration method and the segmentation method becomes robust because in all positions the line is accurately segmented. In addition, the phantom does not suffer from any oscillation problem during the calibration since a well tensioned and firm steel wire is scanned. In perfect vertical position the phantom suffered from reflections of the base plate, this was simply solved by covering the plate by a rubber layer to eliminate them.

The optimization method minimized the squared distances of measured points from a plane passing through the wire line. Since no physical translations were used, differently from Prager et al., ${ }^{12}$ the plane could be any plane 


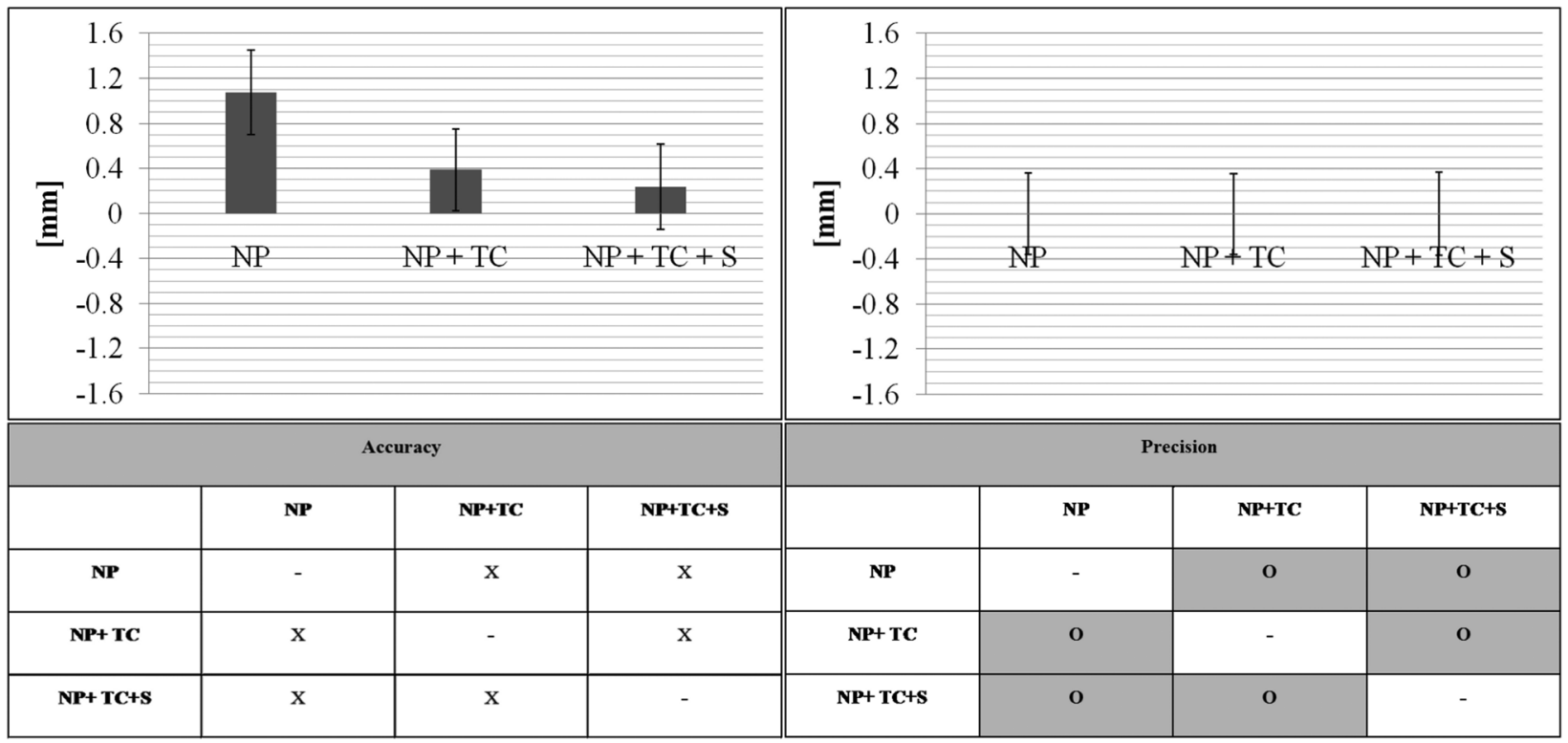

FIG. 7. Calibration method results. On the left, accuracy is shown and on the right, precision is reported. In the bottom tables, test results are shown, " $\mathrm{X}$ " indicates significant variance difference, "o" indicates no significant difference.

passing through the wire line. At each iteration, only two plane parameters are constrained by the optimization, since the rotation of the probe around an axis orthogonal to the plane and applied in the center of the wire and the rotation around the wire do not change the cost figure. We thought that including more constraints could have improved the noise rejection of the algorithm. For this reason, we fixed the other plane parameters adding "virtual displacements" of the probe DRF on a virtual plane. In this way, the plane passing through the wire line is constrained to be the virtual plane. We achieved the same noise rejection as in Prager et al. ${ }^{12}$ performing virtual displacements on a perfect plane, without flatness issues. The results showed that this constraint increased the accuracy of the estimate, although the precision was not improved.

The time required for the calibration is drastically decreased, since the physical translation of the probe along the plane is removed. The NearLab phantom calibration time is constrained by the probe-line alignment, and then the calibration requires less than 4 min to be accomplished.

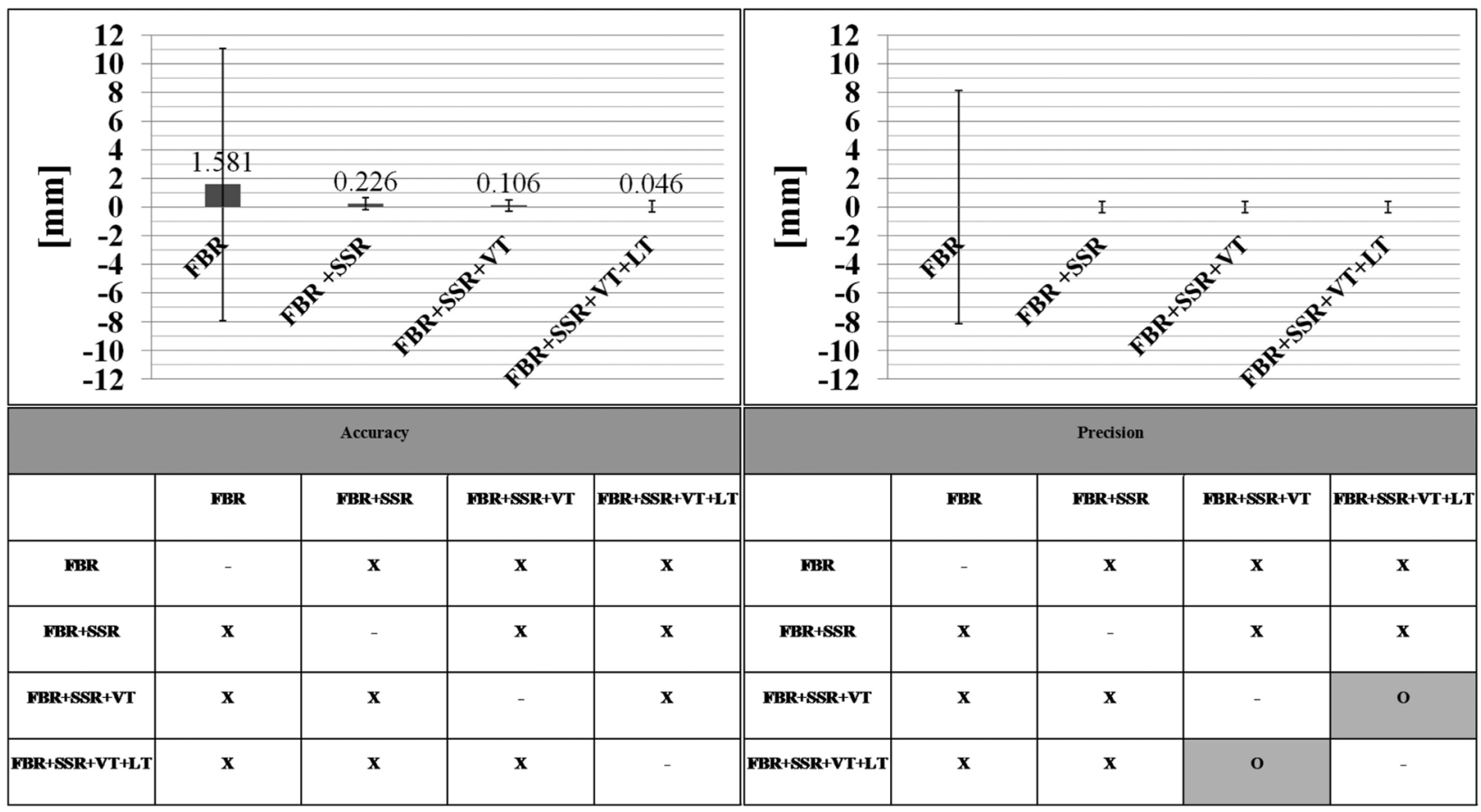

FIG. 8. Calibration protocol results. On the left, accuracy is shown and on the right, precision is shown. In the bottom tables, test results are shown, " $\mathrm{X}$ " indicates significant variance difference, "o" indicates no significant difference. 


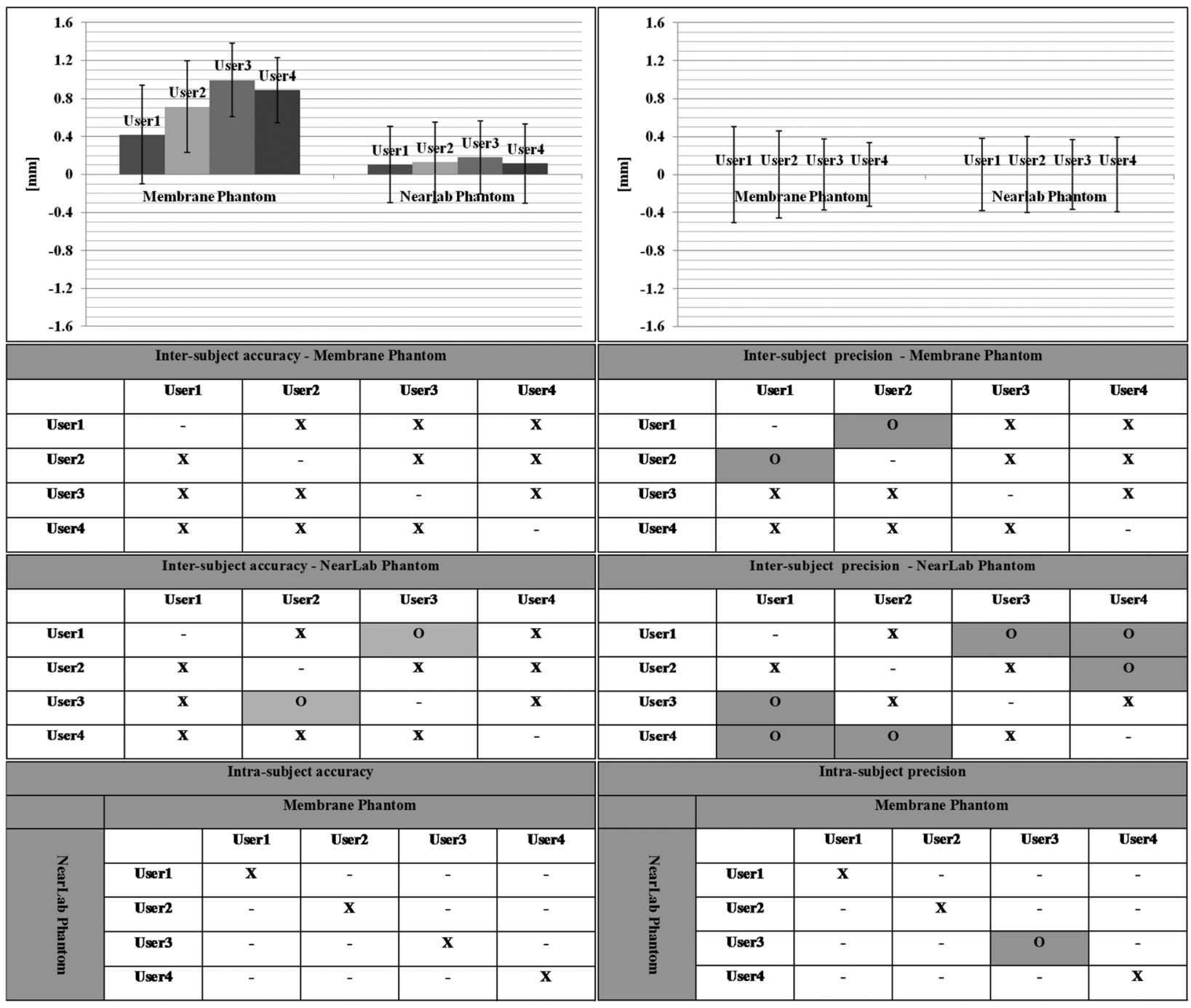

FIG. 9. Intersubject and intrasubject variability results. On the left, accuracy is shown and on the right, precision is shown. At the bottom, test results are shown, "X" indicates variance difference, "o" indicates the variance equality.

The SW framework, which the US acquisition software is part of, is a modular robotic-based system for neurosurgery applications. In this scenario, sensor information redundancy is necessary in order to assure the robotic system safety. In order to assure components interfaceability and to manage the US information by any client computer, the images are processed by a server and then sent to clients through CORBA protocol together with the DRF pose information. The SM is able to simultaneously manage multimodal sensors (optical tracking system, electromagnetic tracking system, US imaging devices). Synchronisation is assured by the SM IGSTKbased architecture, which associates position data and images with timestamps (only $17 \mathrm{~ms}$ of interquartile range shows the delay is approximately constant). Results show that such architecture does not allow, at this stage, freehand realtime or volume reconstruction, since post processing of data are required for proper time alignment. Predictors based on Kalman filtering theory can allow real-time applications. Computing time issues are currently being solved taking advantage of graphics processing units (GPU), as in Dai et al.,39 but imaging prediction is not feasible without segmentation, thus limiting the chances of a strict real-time capability. Nevertheless a slow exploration (few $\mathrm{mm}$ per second) is indeed possible.

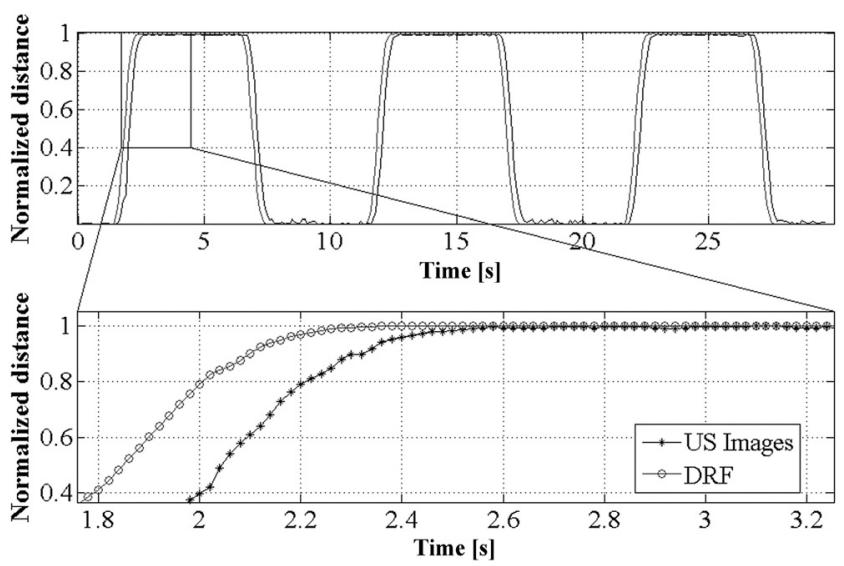

FIG. 10. Top panel, US Image data and position data of the probe DRF versus time. Closer view of the time lag between the two data streams. 
While calibrating, translation movements can be simulated in order to let the optimization algorithm converge more robustly, since the possible plane orientations are fixed already from the first iterations: the calibration algorithm designed proved to decrease the point reconstruction accuracy to $0.2 \mathrm{~mm}$, as mean signed distance value from the real plane, with $0.4 \mathrm{~mm}$ as standard deviation, which is better than the $0.7 \pm 0.6 \mathrm{~mm}$ reported by Hartov et al. ${ }^{25}$ Extensive literature comparison is presented in Mercier et al. ${ }^{11}$ best results in terms of mean point reconstruction precision and accuracy are presented in Prager et al. ${ }^{12}(0.04 \mathrm{~mm}$ as mean precision value, with standard deviation values not reported, and $0.04 \mathrm{~mm}$ as mean accuracy value, with $1.12 \mathrm{~mm}$ of standard deviation). Boctor et al..$^{40}$ obtained $0.6 \mathrm{~mm}$ as mean precision value with standard deviation comparable with our approach, and $0.25 \mathrm{~mm}$ as mean accuracy value, with greater standard deviation $(1.78 \mathrm{~mm})$. Results presented by Kowal et $a l^{41}$ are comparable with ours in terms of accuracy performances, but worse (one order of magnitude) in terms of precision. Recently, Mercier et al. ${ }^{4}$ reported $0.64 \mathrm{~mm}$ as mean value for the point reconstruction accuracy. Chen et al., ${ }^{42}$ who performed beam thickness correction in the calibration, ${ }^{21}$ reached a similar value $(0.66 \mathrm{~mm}$ as mean accuracy).

In our approach, calibration was performed detecting a line in the calibration phantom to avoid the beam thickness problem. ${ }^{12,19,21}$ Accuracy assessment was then performed using a plane. Therefore, the determination of the points on the line in the US image was biased, since the line thickness was changing, according to the orientation of the probe with respect to the plane. For this reason, error data distribution appears to have a non zero bias. Considering the US images resolution $(0.3 \mathrm{~mm})$ and the optical localization system accuracy $(0.15 \mathrm{~mm})$, our error, which satisfies the specification of the surgical application, is mostly due to the measurement systems rather than to the algorithm. Further developments will modify the calibration cost function to minimize the distance between US detected line and a calibration line, getting rid of the "virtual plane".

For accurately calibrating the probe, two rotational movements (around the $X$ and $Y$ directions as shown in Fig. 4) and at least one translational movement are required, confirming what stated in Hsu et al. ${ }^{23}$ The NearLab phantom allows performing such movements in an easy and reliable way. Using the NearLab calibration phantom, besides allowing better calibration performances in terms of mean and standard deviation of the errors, allows also a lower user dependency of the result.

\section{ACKNOWLEDGMENTS}

This work was supported by the EU Project Grant ROBOCAST FP7-ICT-215190. The authors wish to thank João Carlos Dalberto from ITIA-CNR for precious help in manufacturing all the mechanical parts of the phantom. All authors disclaim any financial and personal relationships with other people or organisations that could inappropriately influence (bias) their work. a) Author to whom correspondence should be addressed. Electronic mail: alberto.vaccarella@mail.polimi.it

${ }^{1}$ A. Gronningsaeter, A. Kleven, S. Ommedal, T. E. Aarseth, T. Lie, F. Lindseth, T. Langø, and G. Unsgård, "SonoWand, an ultrasound-based neuronavigation system," Neurosurgery 47, 1373-1380 (2000).

${ }^{2}$ F. Lindseth, J. H. Kaspersen, S. Ommedal, T. Lang $\varnothing$, J. Bang, J. Hokland, G. Unsgaard, and T. A. Nagelhus Hemes, "Multimodal image fusion in ultrasound-based neuronavigation: improving overview and interpretation by integrating preoperative MRI with intraoperative 3D ultrasound," Comput. Aided Surg. 8, 49-69 (2003).

${ }^{3}$ G. Unsgård, O. Solheim, F. Lindseth, and T. Selbekk, "Intra-operative imaging with 3D ultrasound in neurosurgery," Acta Neurochir. Suppl. (Wien) 109, 181-186 (2011).

${ }^{4}$ L. Mercier, R. F. Del Maestro, K. Petrecca, A. Kochanowska, S. Drouin, C. X. Yan, A L. Janke, S. J. Chen, and D. L. Collins, "New prototype neuronavigation system based on preoperative imaging and intraoperative freehand ultrasound: system description and validation,” Int. J. Comput Assist. Radiol. Surg. 6, 507-522 (2011).

${ }^{5}$ P. Wong, T. Muanza, E. Reynard, K. Robert, J. Barker, and K. Sultanem, "Use of three-dimensional ultrasound in the detection of breast tumor bed displacement during radiotherapy," Int. J. Radiat. Oncol., Biol., Phys. 79, 39-45 (2010).

${ }^{6} \mathrm{H}$. Johnston, M. Hilts, W. Beckham, and E. Berthelet, "3D ultrasound for prostate localization in radiation therapy: a comparison with implanted fiducial markers," Med. Phys. 35, 2403-2413 (2008).

${ }^{7}$ D. C. Barratt, C. S. K. Chan, P. J. Edwards, G. P. Penney, M. Slomczykowski, T. J. Carter, and D. J. Hawkes, "Instantiation and registration of statistical shape models of the femur and pelvis using 3D ultrasound imaging," Med. Image Anal. 12, 358-374 (2008).

${ }^{8}$ P. Mozer, A. Leroy, Y. Payan, J. Troccaz, E. Chartier-Kastler, and F. Richard, "Computer-assisted access to the kidney," Int. J. Med. Rob. Comp. Assist. Surg. 1, 58-66 (2005)

${ }^{9}$ J. Bax, D. Smith, L. Bartha, J. Montreuil, S. Sherebrin, L. Gardi, C. Edirisinghe, and A. Fenster, "A compact mechatronic system for 3D ultrasound guided prostate interventions," Med. Phys. 38, 1055-1069 (2011).

${ }^{10}$ D. Ni, Y. Qu, X. Yang, Y. Chui, T. T. Wong, S. Ho, and P. Heng, "Volumetric ultrasound panorama based on 3D SIFT," Presented at the 11th International Conference on Medical Image Computing and Computer-Assisted Intervention-MICCAI, Springer, Berlin/Heidelberg, New York, 2008, September 6-10.

${ }^{11}$ L. Mercier, T. Lang $\varnothing$, F. Lindseth, and L. D. Collins, "A review of calibration techniques for freehand 3D ultrasound systems," Ultrasound Med. Biol. 31, 143-165 (2005).

${ }^{12}$ R. Prager, R. Rohling, A. Gee, and L. Berman, "Rapid calibration for 3D freehand ultrasound," Ultrasound Med. Biol. 24, 855-869 (1998).

${ }^{13}$ D. M. Muratore, and R. L. Galloway, "Beam calibration without a phantom for creating a 3D freehand ultrasound system," Ultrasound Med. Biol. 27, 1557-1566 (2001).

${ }^{14}$ G. M. Treece, A. H. Gee, R. W. Prager, C. J. C. Cash, and L. H. Berman, "High-definition freehand 3D ultrasound," Ultrasound Med. Biol. 29, 529-546 (2003).

${ }^{15}$ P. W. Hsu, G. M. Treece, R. W. Prager, N. E Houghton, and A. H. Gee, "Comparison of freehand 3D ultrasound calibration techniques using a stylus," Ultrasound Med. Biol. 34, 1610-1621 (2008).

${ }^{16}$ P. R. Detmer, G. Bashein, T. Hodges, K. W. Beach, E. P. Filer, D. H. Burns, and D. E. Strandness, Jr., "3D Ultrasonic image feature localization based on magnetic scanhead tracking: in vitro calibration and validation," Ultrasound Med. Biol. 20, 923-936 (1994).

${ }^{17}$ M. J. Gooding, S. H. Kennedy, and J. A. Noble, "Temporal calibration of freehand three-dimensional ultrasound using image alignment," Ultrasound Med. Biol. 31, 919-928 (2005).

${ }^{18}$ F. Lindseth, G. A. Tangen, T. Lang $\varnothing$, and J. Bang, "Probe calibration for freehand 3D ultrasound," Ultrasound Med. Biol. 29, 1607-1623 (2003).

${ }^{19} \mathrm{~T}$. Lang $\varnothing$, "Ultrasound guided surgery: image processing and navigation," Ph.D. thesis, Norwegian University of Science and Technology, Trondheim, Norway, 2000.

${ }^{20}$ F. Rousseau, P. Hellier, and C. Barillot, "A novel temporal calibration method for 3D ultrasound," IEEE Trans. Biomed. Eng. 25, 1108-1112 (2006).

${ }^{21}$ T. K. Chen, R. E. Ellis, and P. Abolmaesumi, "Improvement of freehand ultrasound calibration accuracy using the elevation beamwidth profile," Ultrasound Med. Biol. 37, 1314-1326 (2011). 
${ }^{22}$ A. H. Gee, N. E. Houghton, G. M. Treece, and R. W. Prager, "A mechanical instrument for 3D ultrasound probe calibration," Ultrasound Med. Biol. 31, 505-518 (2005).

${ }^{23}$ P. W. Hsu, R. W., Prager, A. H. Gee, and G. M. Treece, "Rapid, easy and reliable calibration for freehand 3D ultrasound," Ultrasound Med. Biol. 32, 823-835 (2006).

${ }^{24} \mathrm{P}$. W. Hsu, "Free-hand three-dimensional ultrasound calibration," $\mathrm{Ph} . \mathrm{D}$. thesis, University of Cambridge, Cambridge, UK, 2007.

${ }^{25}$ A. Hartov, K. Paulsen, J. Songbai, K. Fontaine, M. L. Furon, A. Borsic, and D. Roberts, "Adaptive spatial calibration of a 3D ultrasound system," Med. Phys. 37, 2121-2130 (2010).

${ }^{26}$ R. W. Prager, A. Gee, and L. Berman, "Stradx: real-time acquisition and visualization of freehand three-dimensional ultrasound," Med. Image Anal. 3, 129-140 (1999).

${ }^{27}$ Q. Huang, Y. Zheng, M. Lu, and Z. Chi, "Development of a portable 3D ultrasound imaging system for musculoskeletal tissues," Ultrasonics $\mathbf{4 3}$, 153-163 (2005)

${ }^{28}$ M. C. Jacobs, M. A. Livingston, and A. State, "Managing latency in complex augmented reality systems," Proceedings of the Symposium on Interactive 3D Graphics, Providence, ACM, New York, NY, Providence, Rhode Island, 1997.

${ }^{29}$ P. W. Hsu, R. W. Prager, A. H. Gee, and G. M. Treece, "Real-time freehand 3D ultrasound calibration," Ultrasound Med. Biol. 34, 239-251 (2008).

${ }^{30}$ E. De Momi and G. Ferrigno, "Robotic and artificial intelligence for keyhole neurosurgery: the ROBOCAST Project, a multi-modal autonomous path planner," Proc. Inst. Mech. Eng. H 224, 715-727 (2010).

${ }^{31}$ A. Vaccarella, P. Cerveri, E. De Momi, and G. Ferrigno, "A new IGSTKbased architecture for the integration of multimodal sensors and robots in neurosurgical robotics applications,” Int. J. Comput. Ass. Radiol. Surg. 5, 308-309 (2010)
${ }^{32}$ W. Emmerich, "Software engineering and middleware: a roadmap," Proceedings of the conference on the future of software engineering, ACM, 2000.

${ }^{33}$ Object Computing Inc., The ACE ORB, www document, http://www. theaceorb.com/, June 2011.

${ }^{34}$ M. A. Fischler and R. C. Bolles, "Random sample consensus: a paradigm for model fitting with applications to image analysis and automated cartography," Commun ACM 24, 381-395 (1981).

${ }^{35} \mathrm{D}$. W. Marquardt, "An algorithm for least-squares estimation of nonlinear parameters,” J. Soc. Ind. Appl. Math. 11, 431-441 (1963).

${ }^{36}$ F. L. Markley, Y. Cheng, J. L. Crassidis, and Y. Oshman, "Averaging quaternions,” J. Guid. Control Dyn. 30, 1193-1196 (2007).

${ }^{37}$ G. R. Sutherland, I. Latour, and A. D. Greer, "Integrating an image guided robot with intraoperative MRI: a review of design and construction," IEEE Eng. Med. Biol. Mag. 27, 529-546 (2008).

${ }^{38}$ C. Nimsky, O. Ganslandt, S. Cerny, P. Hastreiter, G. Greiner, and R. Fahlbusch, "Quantification of, visualization of, and compensation for brain shift using intraoperative magnetic resonance imaging," 47, 1070-1080 (2000).

${ }^{39}$ Y. Dai, J. Tian, D. Dong, G. Yan, and H. Zheng, "Real-time visualized freehand 3D ultrasound reconstruction based on GPU," IEEE Trans. Inf Technol. Biomed. 14, 1338-1345 (2010).

${ }^{40}$ E. M. Boctor, A. Jain, M. A. Choti, R. H. Taylor, and G. Fichtinger, "Rapid calibration method for registration and 3D tracking of ultrasound images using spatial localizer," Proc. SPIE 5035, 521-532 (2003).

${ }^{41}$ J. Kowal, C. A. Amstutz, M. Caversaccio, and L. P. Nolte, "On the development and comparative evaluation of an ultrasound B-mode probe calibration method," Comput. Aided Surg. 8, 107-119 (2003).

${ }^{42}$ T. K. Chen, A. D. Thurston, R. E. Ellis, and P. Abolmaesumi, "A realtime freehand ultrasound calibration system with automatic accuracy feedback and control," Ultrasound Med. Biol. 35, 79-93 (2009). 